\title{
Bioethics Committees: Comparison of Small Community Hospitals and Large Regional Medical Centers
}

\author{
Richard Boudreau* \\ Faculty Loyola Marymount Univ. Bioethics Institute, Faculty UCLA Dept. Oral \& Maxillofacial Surgery, USA \\ *Corresponding author: Richard Boudreau, Faculty Loyola Marymount Univ. Bioethics Institute, Faculty UCLA Dept. Oral \& \\ Maxillofacial Surgery, Attorney at Law, Member Calif. Bar, Wash. DC Bar \& US Supreme Court Bar, USA
}

\begin{abstract}
Though most large regional medical centers and many small community hospitals have bioethics committees, distinct differences between these two types of facilities can impact the purpose and function of these committees. A surge in the creation of these committees since the 1990s has led to studies of the different approaches used by these committees, the different compositions, and the roles that hospital ethics committees (HECs) play in defining standards for end-of-life care. A comparison of small and large facility HECs provides some insight into the challenges in applying ethical standards in the healthcare field.
\end{abstract}

\section{Purpose of HECs}

Both large regional medical centers and small community or rural community hospitals have developed bioethics committees to address some significant issues that are linked to the expansion of medical technology and the ethical treatment of patients both during care and in end-of-life planning. All clinicians and administrators in these facilities, whether large or small, are faced with ethical issues that can have a negative impact on the ability to provide patients with quality care if not addressed properly. Though there are standards of care that are identified under the law and related as a part of professional ethical codes, there are issues that must be more clearly extrapolated in order to fully determine how standards are applied in specific healthcare settings. The main areas related to ethics that are frequently addressed in both small rural hospitals and large medical centers include patient autonomy, confidentiality, fidelity and distributive justice [1]. Different approaches to the management of these issues by HECs can extend from the environments and needs of the population they serve.

\section{Large Regional Medical Center HECs}

Large regional medical centers are more likely to have HECs than small, rural hospitals, and these HECs often take a much larger role in the development of policies, the creation of educational programming for staff, and the assessment of specific cases in regards to the application of ethical standards. Many of these large healthcare facilities apply a set of broad reaching ethical standards that cover a range of situation. Because these facilities often seek out methods to address ethical standards, codes of conduct, and ethics applied to decision-making, these HECs are usually comprised of a multidisciplinary collective of professionals that can shed insight into the application of ethical standards at different levels of the organization [2]. Subsequently, the direction and approach of these HECs are driven by both the perspectives of departmental representatives and changing needs for the health care organization. It is not surprising that large facility HECs have had to modify their ethical standards in relation to changing current technological advancements and anticipated changes over time.

\section{Small HECs}

Small hospitals, especially those that serve rural populations, experience unique issues related to the ethical standards of care for patients and the use of HECs. First, not all small hospitals have HECs, and not all small hospitals share the same focus on broad reaching ethical standards that seem a necessity in large healthcare settings. In addition, while large healthcare institutions' HECs reflect the diversity of services and the range of providers in the setting, small facilities often have much smaller levels of representation in HECs [3]. While hospitals can apply standards with a high degree of equanimity, small hospitals often experience struggles because of lack of resources, overlap between personal 
and professional relationships, and culturally distinct needs of the patient population. These small rural hospitals, though, meet the healthcare needs of more than 60 million people in this country each year, or over one quarter of the total population [4]. The context of these communities not only shapes the perspectives of HEC participants, but also of the people for whom ethical standards and subsequent healthcare policies are developed. The combination of social, geographical, cultural, and religious elements that impact that process of care can have an influence on the way in which ethical standards, professional codes, and end-of-life decisionmaking are applied to daily operations. In a study of small hospital ethics committees, ethical policies developed as an extension of HEC operations were somewhat less broad reaching and allowed for a higher degree of interpretation on the part of health care professionals. This was due in part to the small size of the facilities and also to the ability of committee members to participate in regular dialogue in an effective manner. Larger hospitals suffer from a level of bureaucracy and poor communications that can determine variability in the effectiveness of ethics committee policies. Rural community hospitals vary from larger health care facilities because they have limited economic resources, lower levels of overall health in patient populations, shared values, limited availability of services and access issues, and overlapping relationships between professionals and patients that can result in a high degree of health provider stress.

\section{General Comparisons}

While large healthcare facilities often share information about approach and standards introduced within the scope of ethical policies and decision-making in the hospital setting, rural hospitals often have unique characteristics that can make it difficult to apply a single set of ethical standards [5]. The Coalition for Rural Health Care Ethics created some working definitions of health care in this setting and determined some of the challenges in meeting the goals of a geographically and socially dispersed population. Both large and small hospitals have similar goals in the use of HECs, including enhancing the quality of patient care and reducing litigation resulting from questions of ethical conduct in patient treatment.

\section{End of Life Care}

In both small and large medical centers, there has been a distinct shift in the approaches to end-of-life care and decisionmaking, which has placed decision-making in the hands of patients and their loved ones or designated surrogates. In the presence of advances in medical technologies and the ability of medical professionals to sustain life, legal regulations about how to proceed in the end-stage of disease provides distinct complications and can involve HECs. When a person has an advanced directive, something that is generally pursued as a part of practices in large hospitals and may be less frequently applied in smaller facilities, the legal regulations about how to proceed are clear. One of the concepts that come into play as a part of the evaluations of HECs in both small and large medical facilities is the concept of medical futility [6]. Medical futility is often discussed in relation to the balance between medical treatment and the reality of human mortality: in every human life, there is a time at which the pursuit of life is no longer either medically prudent or in the best interest of the patient. When treatments can no longer result in a person being autonomous, the termination of medical services is viewed as an ethically sound choice. Even in the presence of life-sustaining technologies, decisions sometimes have to be made that "enough is enough."

The issue of medical futility has not only brought up discussions about mortality and the nature of medical interventions, but also the legal parameters of medical care and the ethical obligations of medical professionals. If a doctor perceives the continuation of treatments as medically futile and discontinues those treatments even in the presence of opposition by the surrogate decisionmakers, one question that may be considered within the scope of HEC functions is if the doctor crossed a line. Subsequent to this, HECs may determine who is responsible for making decisions regarding the cessation of treatment and can also determine if a physician can be held legally responsible for decisions about termination of treatments. Both large and small medical centers have recognized that there are distinct challenges in end-of-life decision-making that create challenges for both families and practitioners. It has been argued that this kind of problem extends from the level of technology that has emerged in end-of-life care and the problems that practitioners face when making decisions about whether to continue with care [7]. Theorists like Robert Burt have argued that there are distinct ethical issues in end-of-life decision-making that can impact how practitioners perceive their roles [8]. While Burt argued that no practitioner should make choices that inflict death on another person, there is a fine line in defining causation when a person's life support systems are shut off by a practitioner. These types of considerations and assessments of behaviors, as well as the policies that direct those behaviors, are the function of HECs [9].

\section{Conclusion}

Both large healthcare facilities and small rural hospitals utilize HECs to help define the protocols and policies related to the fine line between medical capabilities and end-of-life decisions. Both of these types of organizations face similar challenges when endof-life care decisions are being made. For large HECs, there may be a greater effort to clearly define and outline protocols that are used in the hospital setting and educate staff to comply with the ethical standards developed. Small hospitals are less likely to have HECs, but when they do, they are more effective in communicating the best approaches to addressing patient needs. Both types of organizations struggle with addressing the legal, social, ethical and cultural ramifications of end-of-life decision-making in the healthcare setting. 


\section{References}

1. Cook A, Hoas H (2008) Ethics and rural healthcare: What really happens? What might help? The American Journal of Bioethics 8(4): 52-56.

2. Curtis J, Burt R (2010) Futility in the intensive care unit: Hard cases make bad law. Critical Care Medicine 38(8): 1742-1743.

3. Gonsoulin T (2009) A survey of Louisiana hospital ethics committees. The Laryngoscope 119(2): 330-340.

4. Jackson E, Olive K (2009) Ethics committees in small, rural hospitals in East Tennessee. Southern Medical Journal 102(5): 481-485.

5. Nelson W (2004) Addressing rural ethics issues. The characteristics of rural health care settings pose unique ethical challenges. Healthcare Executive 19(4): 36-37.
6. Nelson W, Greene M, West A (2010) Rural healthcare ethics: No longer the forgotten quarter. Cambridge Quarterly of Healthcare Ethics 19(4): 510-517.

7. Howard K, Bushy, A (2007) A proposed rural healthcare ethics agenda Journal of Medical Ethics, 33(3): 136-139.

8. Rich B (2007) Causation and Intent: Persistent Conundrums in End-ofLife Care. Cambridge Quarterly of Healthcare Ethics 16: 63-73.

9. Schneiderman L (2011) Defining medical futility and improving medical care. Journal of Bioethical Inquiry 8(2): 123-131.

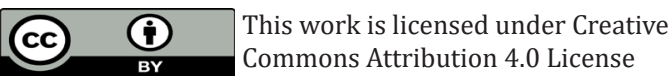

To Submit Your Article Click Here:

Submit Article

DOI: $10.32474 /$ RRHOAJ.2020.05.000212

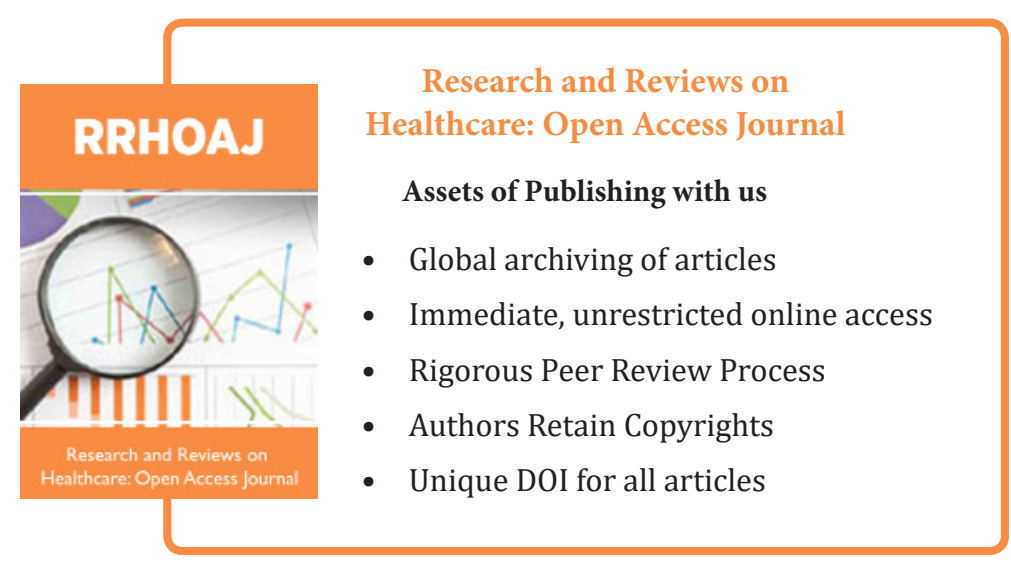

